\title{
Desarrollo de un proceso de obtención de un pistón de 100 cc de dos tiempos mediante ingeniería inversa
}

DOI: https://doi.org/10.33262/ap.v3i3.70

\begin{abstract}
(c) (i) (ㅇ)
Development of a process for obtaining a two-stroke 100cc piston by means
\end{abstract}

Edison Patricio Abarca Pérez. ${ }^{1}$, Víctor David Bravo Morocho. ${ }^{2}$, Jose Luis Cayancela Achote. ${ }^{3}$ \& Luis Fernando Buenaño Moyano. ${ }^{4}$

\begin{abstract}
.
Introduction. In our country, there is a lack of spare supply, in particular for motorcycle engine stroke pistons, since the production of this element only occurs in countries such as: Germany, Argentina, Spain, Turkey, Great Britain, and the United States, Brazil, Japan, China and India, these being the pioneers that supply the replacement stock both worldwide and in Latin America. Objective. Obtain a process for obtaining a two-stroke $100 \mathrm{CC}$ piston by applying reverse engineering from a commercial piston through the use of different tests such as: spectrometry, hardness test and micrography. Methodology. To obtain it, it has been made from recycled pistons as a source of raw material, also contributing to the environment by reusing pistons that are out of service, on the other hand, for the sand mold casting process It has based on standards and previous research to reduce effects such as excess porosity, fractures, etc., since they can influence the final product. After this, after obtaining the piston, by means of the aforementioned tests, a

\footnotetext{
1 Escuela Superior Politécnica de Chimborazo, Facultad de Mecánica, Riobamba, Ecuador. edison.abarca@espoch.edu.ec, https://orcid.org/0000-0001-7041-4805X

2 Escuela Superior Politécnica de Chimborazo, Facultad de Mecánica, Riobamba, Ecuador. victor.bravo@espoch.edu.ec, https://orcid.org/0000-0001-5629-259X

3 Escuela Superior Politécnica de Chimborazo, Facultad de Mecánica, Riobamba, Ecuador. jcayancela@espoch.edu.ec, https://orcid.org/0000-0003-3560-021X

4 Escuela Superior Politécnica de Chimborazo, Facultad de Mecánica, Riobamba, Ecuador. lfbuenanio@espoch.edu.ec, https://orcid.org/0000-0002-2194-4102X
} 
comparison is made between the obtained and the original, additionally with a tensile test, providing data such as the ultimate tensile stress and the yield point. Results. In this way, a functional piston was obtained, and it is concluded that during casting it is important to consider that silicon by the action of temperature decreases its percentage by mass, an important factor since as a chemical element it is difficult to find in the market.

Keywords: Alloys, Casting, Reverse Engineering, Piston, Mechanical Properties

\section{Resumen.}

Introduccion. En nuestro país se ve la falta de abastecimiento de repuesto en particular los pistones de tiempos para motor de motocicletas, dado que la producción de este elemento solo se da en países tales como: Alemania, Argentina, España, Turquía, Gran Bretaña, Estados Unidos, Brasil, Japón, China e India, siendo estos los pioneros que abastecen el stock de recambio tanto a nivel mundial como en Latinoamérica. Objetivo. Obtener un proceso de obtención de un pistón de $100 \mathrm{CC}$ de dos tiempos aplicando la ingeniería inversa a partir de un pistón comercial mediante el empleo de diferentes ensayos como: espectrometría, ensayo de dureza y micrografía. Metodología. Para la obtención de la misma se ha elaborado a partir de pistones reciclados como fuente de materia prima, contribuyendo además con el medio ambiente al reutilizar los pistones que están fuera de servicio, por otra parte, para el proceso de fundición en molde de arena se ha basado en normas e investigaciones previas para reducir efectos como el exceso de porosidad, fracturas, etc., puesto que pueden influir en el producto final. Posterior a ello tras obtener el pistón, mediante los ensayos mencionados se realiza una comparación entre el obtenido y el original, adicional con un ensayo de tracción aportar con datos como el esfuerzo ultimo de tracción y el límite de fluencia. Resultados. De esta manera se obtuvo un pistón funcional además se concluye que durante la fundición es importante considerar que el silicio por acción de la temperatura disminuye su porcentaje en masa, factor importante ya que como elemento químico es difícil de encontrar en el mercado.

Palabras claves: Aleaciones, Fundición, Ingeniería Inversa, Pistón, Propiedades Mecánicas

\section{Introducción.}

En diferentes En diferentes puntos de venta de stock de partes de recambio para motocicletas, en el Ecuador desde hace algunos años atrás, se ha dejado de abastecer de algunos elementos, entre ellos se tiene a los pistones para motores de dos tiempos, esto en vista de que la mayor parte de atención se ha centrado en importar elementos de mantenimiento para vehículos en general con constan de motores de cuatro tiempos. Por tanto, en el Ecuador se registra una gran demanda de repuestos para motores de dos tiempos, con la finalidad de prolongar la vida útil del mismo, tomando en cuenta los límites permisibles de contaminación. 
La producción masiva de pistones se halla en países tales como: Alemania, Argentina, España, Turquía, Gran Bretaña, Estados Unidos, Brasil, Japón, China e India, siendo estos los pioneros que abastecen el stock de recambio tanto a nivel mundial como en Latinoamérica. (MAHLE Metal Leve S.A., 2020)

En consecuencia, Ecuador es uno de los países con más importaciones de pistones de dos tiempos para motores. El pistón es el elemento móvil principal en el funcionamiento de un motor de combustión interna, se desgasta característicamente reduciendo el diámetro de su perfil debido a la fricción producida, de tal forma que aumentando la holgura en el alojamiento de los anillos; y tras haber cumplido la vida útil, se ve en la obligación de sustituirlo por un repuesto con características similares, garantizando de esta manera prolongar la vida útil de dicho motor, por lo tanto, el pistón se torna en un elemento de recambio frecuente. (Crouse, 1992)

Toda importación está sujeta a su respectivo valor arancelario en función del número de cupos permitida en el Ecuador, siendo a la vez analizado y distribuido de tal manera que en Ecuador se prioriza las importaciones de autopartes para vehículos, existiendo menor interés por los pistones de dos tiempos empleados en motores de motocicletas. (COMEX, 2013)

Por tal motivo se obtendrá un proceso de obtención de pistones de dos tiempos para motores de $100 \mathrm{CC}$ siendo escogido este modelo para un estudio por ser el más comercial en el medio y además contribuir en el proyecto de investigación "Desarrollo de un prototipo de motor de dos tiempos mediante la integración de las herramientas CADCAM-CAE", teniendo en cuenta las características necesarias para que el mismo sea un elemento de recambio óptimo y garantizar la funcionabilidad de este elemento en un motor.

\section{Materiales de un pistón}

Generalmente los pistones están constituidos de una aleación de aluminio-silicio, con un contenido de silicio mínimo del $12 \%$, se añaden además pequeñas cantidades de otros elementos como: cobre, hierro, cobre, níquel, entre otros. Todos estos materiales, mediante estudios persiguen un fin común que es aligerar el producto resultante, se puede obtener ya sea mediante forja o fundición (Mackay, 2016).

La aleación aluminio silicio es muy ligero y altamente resistente, pueden ser sometidos a diferentes tratamiento de templado posterior a la fundición para mejorar sus propiedades de dureza y resistencia al desgaste, por otra si al silicio se puede añadir hasta un $25 \%$ del valor mínimo al alear con el aluminio para aumentar las propiedades de dureza, disminuir el desgaste y disminuir la dilatación térmica ya sea en motores por argumentos de preparación, altas solicitaciones como lo es motores Diesel sobrealimentados o motores de dos tiempos como de motocicletas (Sanz, 2007; citado en Guarderas 2013, p. 69). 


\section{Aleaciones de aluminio}

El aluminio es un metal no ferroso que más abunda sobre la corteza terrestre principalmente formando silicatos y en forma de óxido debido a su alta afinidad con el oxígeno, con un $8,07 \%$ de peso estimado, por otra parte también está el silicio con el 27,69 \%, oxigeno con el 46,71\%, Hierro con el 5,05\%, Magnesio con el 2,08\%, siendo estos algunos elementos químicos que conforman parte de las aleaciones más comunes con el aluminio en el campo automotriz, por ser versátil y económicos, además presentando propiedades como: resistencia a la corrosión, relación elevada peso resistencia, baja densidad (Polmear et al., 2017, pp. 7-9).

La fabricación de pistones se puede clasificar en dos grupos quo son: aleaciones de aluminio para forja o conformado (Polmear, 1997) y aleaciones de aluminio para fundición (Zolotorevsky, 2007).

\section{Aleaciones de Aluminio para Fundición}

Este tipo de aleación comprende más del $90 \%$ de todas las fundiciones de aluminio a nivel general, pero respecto a la fabricación de piezas automotrices fundidas se estima que solo está entre el 20 al 30\% en la producción usando este método para obtener estos elementos, de los productos de aluminio fabricado por este método, se mantiene hasta la fecha por su excelente capacidad de moldeo, resistencia a la fisuración y otras propiedades tecnológicas que son aplicables para casi todos los métodos de fundición (Zolotorevsky, 2007),

\section{Aleaciones de Aluminio - Silicio}

Las aleaciones de Aluminio - Silicio $(\mathrm{Al}-\mathrm{Si})$, se los denomina también como aleaciones binarias siendo este el más común en un $90 \%$ del total de partes producidas de aleación de aluminio por fundición, estos prestan propiedades tales como: excelente fluidez, capacidad de fundición, resistencia a la corrosión y una fácil capacidad de moldeo, de tal modo que es importante para el desarrollo en el área automotriz para la fabricación de partes como: pistones, cajas de cambio, cabezotes, bloques de motor, desalojando a las fundiciones tradicionales de hierro (Askeland, 2016)

El incremento de silicio aumenta la dureza reduciendo la maquinabilidad, porcentaje de elongación, la expansión térmica e incrementa la resistencia última a la tracción (UTS), pero para adquirir ciertas propiedades especificas en el ámbito automotriz se suele añadir otro elemento aleante como el hierro, magnesio, cobre, manganeso entre los principales para dicho propósito, otra característica importante es la baja densidad del silicio que es $\left(2.34 \mathrm{~g} / \mathrm{cm}^{\wedge} 3\right)$, este aporta la reducción al peso total de la aleación. Mediante el sistema de designación de la Asociación de Aluminio, las aleaciones se hallan en la serie 4xxx que corresponde al caso de aleaciones de forja y en el 3xx.x para aleaciones de fundición que son el objeto de estudio del presente trabajo (Davis, 2007) 


\section{Diagrama de fase de Al-Si}

Los diagramas de fase permiten conocer: qué fases se forman, a qué temperatura se forman las fases, las cantidades relativas de cada fase, la composición de las fases y cómo se distribuyen los elementos solutos entre las fases y cuán difícil o fácil será colocar un elemento de aleación específico en aluminio, las mismas que están descritas mediante gráficas del estado físico de una o más substancias bajo diferentes condiciones de presión y temperatura. Estos diagramas permiten conocer: las fases que se forman a determinadas condiciones, la temperatura de formación de las fases, la cantidad relativa de cada fase, la composición de cada fase y su solubilidad a diferentes temperaturas (Sigworth, 2013)

"El sistema aluminio-silicio es un eutéctico binario simple con solubilidad limitada de aluminio en silicio y solubilidad limitada de silicio en aluminio. Solo hay una reacción invariante en este diagrama, a saber, $\mathrm{L} \rightarrow \alpha+\beta$ (eutéctico)" (Nayak $\&$ Karthik, 2001, p. 8)

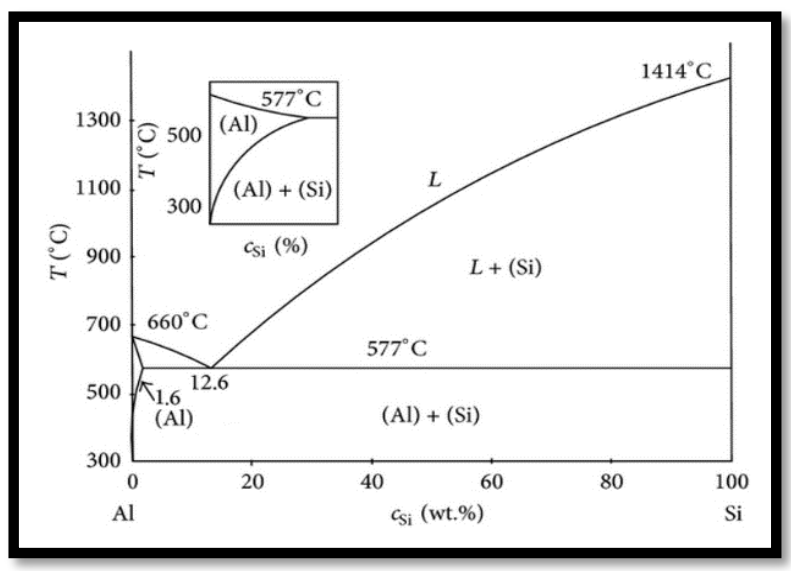

Gráfico 1. Diagrama de fase del sistema binario Al-Si Fuente: (Nayak \& Karthik, 2001, p. 8)

Para la fusión de Al y Si se encuentran a una eutéctica, con una composición de 12.6 por ciento en peso de Si y una temperatura de $577^{\circ} \mathrm{C}$.

La solubilidad máxima es 1,65 por ciento en peso a la temperatura eutéctica, el silicio se disuelve en aluminio.

\section{Aleaciones hipoeutécticas}

Estas aleaciones tienen un contenido de silicio menor que la composición eutéctica. La mayoría de las aleaciones hipoeutécticas comunes tienen entre 5\% y 9-10\% de silicio, estas aleaciones están diseñadas principalmente para aplicaciones de alta resistencia donde también se requiere buena ductilidad (Sigworth, 2013, p. 2).

\section{Aleaciones eutécticas}

Estas aleaciones tienen entre 10 y $13 \%$ de silicio, y consisten principalmente en $\mathrm{Al}-\mathrm{Si}$ eutéctico en la estructura del molde. Tienen un rango de congelación estrecho, excelente fluidez y son fáciles de moldear. También tienen buena resistencia al desgaste y son 
bastante dúctiles cuando no están aleados y tratados térmicamente con alta resistencia. Este tipo de aleaciones son usadas para la fabricación de pistones, muchos de ellos cuentan con otros elementos aleantes como: $\mathrm{Cu}, \mathrm{Mg}$ o $\mathrm{Ni}$, que da las características específicas del pistón (Sigworth, 2013, p. 2).

\section{Aleaciones hipereutécticas.}

Estas aleaciones tienen entre 15 y $20 \%$ de silicio, por lo que su estructura de fundición está compuesta de partículas de silicio primarias incrustadas en una matriz de $\mathrm{Al}-\mathrm{Si}$ eutéctico. Estos materiales tienen una resistencia al desgaste notable y se usan donde se desea esta característica: para pistones, bloques de motor sin revestimiento y piezas de compresores (Cao \& Campbell, 2003).

\section{Metodologia.}

La metodología de la investigación de acuerdo a las posibilidades del investigador y según el tema de investigación elige la metodología adecuada que conlleve a un enfoque claro, mediante un orden lógico y un procedimiento riguroso para dar solución a un problema planteado validando así la investigación.

\section{Diseño CAD}

El proceso de diseño hoy en día se convirtió en la base para el diseño de piezas y componentes que contribuyeron al desarrollo en el sector automotriz, utilizando complejas herramientas que facilitan el proceso y desarrollan el diseño, además de funciones como cálculos y simulaciones, etc. (Domínguez y Espinosa, 2002; citado en Díaz 2013, p. 3).

\section{SOLIDWORKS}

Permite realizar planos en 2D, modelar piezas en 3D y el ensamblaje respectivo de las piezas modeladas, también cuenta con herramientas adicionales para obtener datos de una simulación, este software posee un abanico de formatos de extensión para los archivos que genera que facilita la interacción entre uno o varios softwares CAD o CAM (Díaz, 2013).

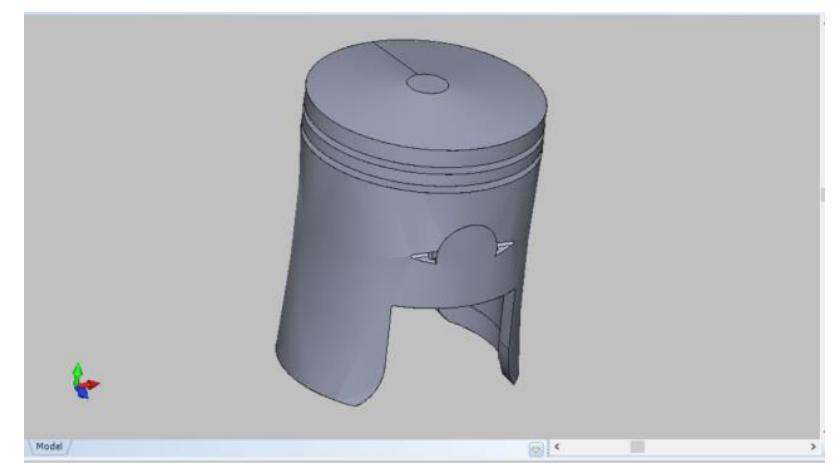

Figura 1. Modelado de pistón en software SolidWorks Fuente: Autor 


\section{Prototipo del pistón - Impresión 3D}

La impresión 3D, conocida como fabricación aditiva, toma un modelo que ha sido previamente procesado por software CAD utilizando los materiales adecuados (PLA) que soporta la impresora al momento de imprimir y lo crea capa por capa, formando un elemento en 3 dimensiones con mucha precisión. La creación de prototipos es una ventaja porque puede identificar fallas, permitiendo aplicar diseños alternativos y asegurarse de tener el mejor producto final después de crearlo.

\section{Obtención de Materiales}

Reciclar los pistones sin usar es una de las maneras para obtener materiales como aluminio y silicio así, de esta manera obtener la materia prima para cada proceso de fundición por lo tanto se considera una opción para su reutilización, y se sabe que el silicio como elemento químico es difícil de obtener en el medio ambiente, aunque existe como una aleación con otros elementos, generalmente tiene un pistón en pequeñas cantidades, por otro lado también en circuitos electrónicos en cantidades relativamente bajas

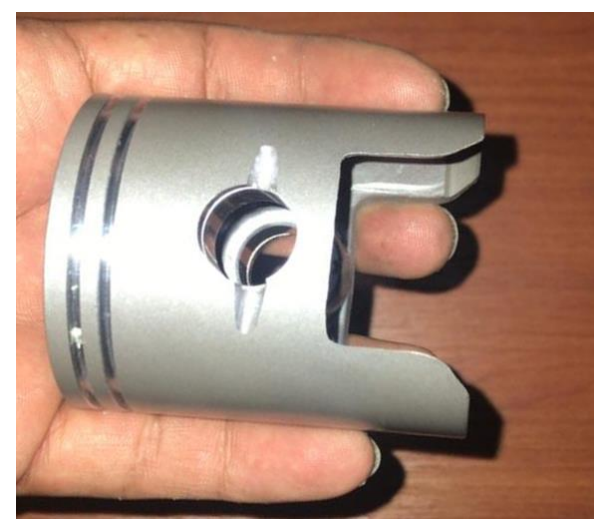

Figura 2. Pistón de dos tiempos Fuente: Autor

\section{Proceso de fundición - Preparación de la Arena}

Una de las características que debe tener la arena es que logre soportar altas temperaturas, es decir, debe tener buenas propiedades refractarias sin que se funda o sufra degradación de cualquier tipo, para ello es importante la granulometría o tamaño de grano de la arena que va entre $1 / 16$ a $2 \mathrm{~mm}$ de diámetro, sin embargo, en ocasiones se realiza un proceso de cernido para obtener la arena mencionada mediante una zaranda de malla metálica de inter - espaciado máximo de $2 \mathrm{~mm}$. Con esta arena, para el proceso de fundición se mezcla de tal modo que contenga (en volumen) $90 \%$ arena, $3-5 \%$ de agua y $7-9 \%$ de arcilla, esperando tener humedad homogénea, de aquí donde aparece las clasificaciones del tipo de arena para fundición (Torres, 2019).

\section{Moldes de Arena}

El molde de arena más empleado para la elaboración de pistones es de tipo dividido, conocido también como molde desechable, con cierta característica de que se puede 
volver a reutilizar la arena que fue empleada durante la obtención de la pieza, este tipo de molde es común para una producción media de elementos, siendo ampliamente usado de muchas maneras según sea la necesidad del medio (Valverde, 2012).

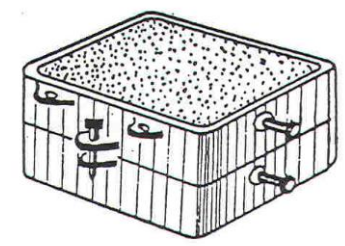

Figura 3: Molde de arena

Fuente: (Valverde, 2012)

\section{Colada}

Con la materia prima lista se dispone a someter a altas temperaturas, sobre los $900{ }^{\circ} \mathrm{C}$. se lo realiza en un horno especialmente diseñado para realizar esta acción, en el presente caso se tiene a disposición pistones desechados que son previamente cortados en pequeños pedazos para obtener la colada como se muestra en la figura 4. Esta colada debe estar completamente en estado líquido para que pueda tener facilidad de ingreso por el conducto de alimentación en el molde y cubrir absolutamente las cavidades que conforma el elemento, en este punto es importante mencionar que las paredes de las cavidades deben estar completamente sólidos para evitar desmoronamientos y por otro lado reducir la probabilidad de generar porosidad en el elemento (Montano, 2011, pp. 44$50)$.

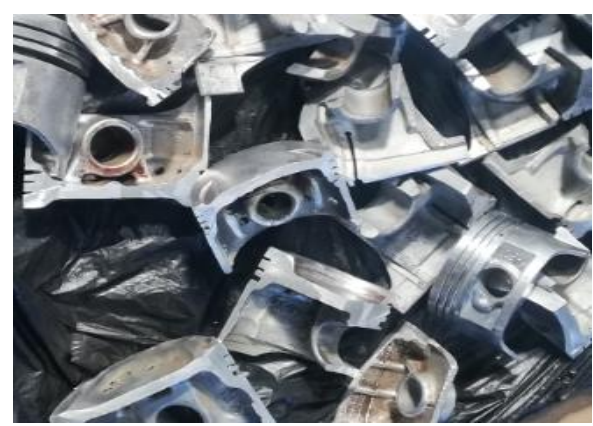

Figura 4: Pistones cortadas en secciones pequeñas

Fuente: Autor

\section{Los sistemas de colada}

Este sistema consta de un conjunto de canales que conducen la aleación líquida hacia la cavidad del molde para su posterior llenado de la colada, dependiendo de su configuración del molde, así pueden ser: colada por la línea de partición del molde, colada por el fondo o sifón, colada escalonada o por etapas y colada directa o por lluvia, siendo este último empleado en el presente estudio (Serrano, 2011).

\section{Mazarota}

Una mazarota es una prolongación de la pieza cuya función es de mantener una reserva aleación líquida, mismo que se emplea en el proceso de solidificación puesto que debe 
compensar las pérdidas de volumen que resulten de las diversas contracciones que experimenta la aleación (Kalpakjian \& Schmid, 2008).

En una fundición se considera la implementación de una o más mazarotas en función del tamaño del producto a obtener y el sistema de colada aplicado, sin embargo, en fundiciones pequeñas el bebedero cumple la función de mazarota si su sistema de colado es directa sobre la cavidad de vaciado (Serrano, 2011).

\section{Bebedero}

El bebedero es un canal cónico vertical mediante el cual se realiza el vaciado de la colada del metal fundido que fluye hacia abajo por acción de la gravedad en el interior del molde. Este se conecta hacia los canales de alimentación en caso de tenerlo caso contrario se conecta directamente con la cavidad de vaciado de la pieza a obtener y suple a la vez la función de una mazarota, y esta debe obedecer a la ecuación 1 (Kalpakjian \& Schmid, 2008).

$$
\frac{A_{1}}{A_{2}}=\sqrt{\frac{h_{1}}{h_{2}}}
$$

Donde A es el área, h representa la altura, subíndice 1 es la parte superior y 2 la parte inferior.

\section{Velocidad del metal fundido}

La velocidad del metal fundido, considerando de que en la parte superior del bebedero sea una sección más grande, se puede determinar la velocidad con la que llega a la parte inferior del bebedero, se empresa mediante la ecuación 2 (Kalpakjian \& Schmid, 2008).

$$
v=\sqrt{2 g h}
$$

Donde g es la gravedad y h representa la altura.

\section{Gasto Volumétrico}

El gasto volumétrico obedece a la ley de la continuidad que enuncia que la tasa de flujo volumétrico va permanecer constante a través del líquido, por lo que va ser igual al producto de la velocidad y su área de la sección transversal del líquido que fluye, expresada así (Groover, 2007):

$$
Q=v_{1} A_{1}=v_{2} A_{2}
$$

Donde Q es el Gasto volumétrico, v representa el volumen y A es el área.

\section{Tiempo de llenado}

Es el tiempo que emplea para llenar tras haber cruzado la base inferior del bebedero por lo que el gasto volumétrico se mantiene constante a través del paso hacia la cavidad al 
igual que en la base, en consecuencia, se puede estimar el tiempo que se requiere para el llenado de la cavidad de un molde de volumen V, y se expresa así (Groover, 2007):

$$
T_{M F}=V / Q
$$

Donde $T_{M F}$ corresponde al tiempo en segundos de llenado en el molde; $V$ es el volumen en centímetros cúbicos de la cavidad y $\mathrm{Q}$ es elgasto volumétrico

\section{Tiempo de solidificación}

Puede ser la fusión de un metal puro o una aleación, y el tiempo de endurecimiento es el tiempo entre la finalización del vertido y la solidificación. El tiempo depende del tamaño y la forma como se vierte. Los cálculos pueden basarse en la ley de Chvorinov, que enuncia (Groover, 2007):

$$
T_{T S}=C m\left(\frac{V}{A}\right)^{2}
$$

Donde $\mathrm{Cm}$ es la Constante de molde $\left[\mathrm{min} / \mathrm{cm}^{2}\right], \mathrm{V}$ es el volumen de la pieza y A es el área.

\section{Ensayo de micrografía}

Mediante la microestructura se puede determinar las fases, su estructura, los constituyentes y sus aleaciones de los metales se pueden determinar bajo observación mediante el microscopio electrónico de barrido o microscopio óptico. Por eso, su análisis es importante para revelar las propiedades específicas de un material. El trabajo actual se centra en medir las fases intermetálicas del aluminio - silicio (Romming et al., 1993).

Preparación de la muestra

Dado que la muestra se basa en la norma ASTM E8, lo más recomendado para una fundición de aleación debe ser una sección transversal perpendicular a la superficie. Esto le permite ver la estructura de principio a fin y considerar las dimensiones. Como se muestra en la Figura 5, con un diámetro de 12 a $25 \mathrm{~mm}$ y una altura considerable que se pueden rectificar fácilmente mediante el pulido (Mendoza Ventura, 2017).

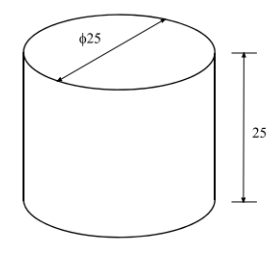

Figura 5: Pistones cortadas en secciones pequeñas Fuente: (Mendoza Ventura, 2017)

\section{Espectrometría}

A través de una espectrometría nos facilita el reconocimiento positivo de materiales presentes en una aleación, generalmente de cobre, silicio, aluminio, hierro, entre otros, 
por otra parte, es un procedimiento que permite desarrollar una ingeniería inversa misma que es el caso de este estudio, para generar un producto nuevo con características propias y determinar la eficiencia del mismo (Q2 ION, 2020, p. 2).

Para ello se requiere de un espectrómetro de emisión óptica por chispa, en este caso es de a marca BRUKERION mostrada en la figura 6, la muestra a utilizar deberá ser preparada para ser ubicada en la cámara de chispeo, para ejecutar el análisis correspondiente, y mostrar los resultados representados (\% elementos) de cada elemento que forma la aleación (Q2 ION, 2020, p. 2).

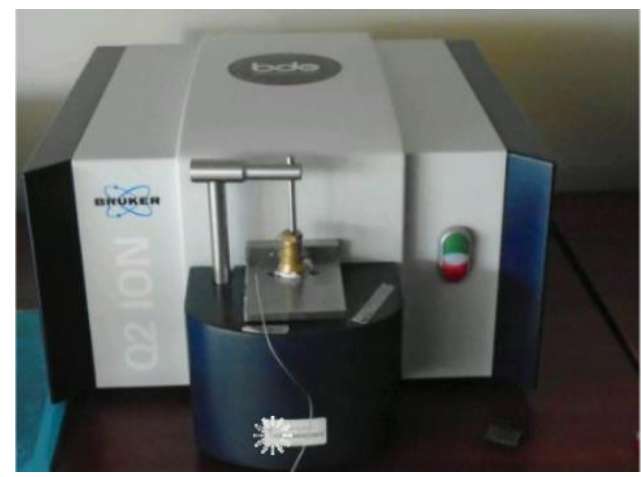

Figura 6: Equipo para espectrometría Q2 ION Fuente: Autor

Según la norma ASTM E 415 el tamaño de la muestra recomendado es de $10 \mathrm{~mm}$ a $38 \mathrm{~mm}$ estas dimensiones pueden variar dependiendo de la disponibilidad o en muchos casos del elemento que sea sometido a evaluación, mientras que para la preparación de la superficie de la muestra es la misma descrita para el ensayo de micrografía antes mencionado (Córdova, 2018).

\section{Ensayo de Dureza Brinell}

Conocida también por dureza a la resistencia a la penetración, abrasión, deformación o destrucción superficial de un material, al estar sometido a esfuerzos externos de otro cuerpo con una superficie más dura. Por otra parte se define también como la capacidad de un material de absorber energía de un esfuerzo justo antes de sufrir una ruptura (ASTM E10, 2017)

$\mathrm{El}$ número de la dureza de Brinell se relaciona directamente con la carga $\mathrm{P}$ que ejerce el indentador de un diámetro determinado D, sobre el área superficial de la huella (ASTM E10, 2017).

$$
\begin{gathered}
H B S \text { ó } H B W=\frac{F}{A} \\
H B S \text { ó } H B W=\frac{F}{\frac{\pi D}{2}\left(D-\sqrt{D^{2}-d^{2}}\right)} \\
H B S \text { ó } H B W=\frac{2 F}{\pi D\left(D-\sqrt{D^{2}-d^{2}}\right)}
\end{gathered}
$$


Para este ensayo se basa en ASTM E 10 (Standard Test Method for Brinell Hardness of Metallic Materials), que lo define de dos maneras, el primer caso se denomina HBS si se usa un indentador de bola de acero, mientras que si usa un indentador de bola de carburo de tungsteno lo han denominado como HBW (ASTM E10, 2017).

\section{Lectura de huella en el micrómetro}

Para obtener la medida marcado por el indentador sobre la probeta se emplea un Microscopio micrómetro de marca Mutitoyo, tipo TM-100 con tolerancia de 0,005 mm, mismo que tiene dos grados de libertad de desplazamiento, horizontal y vertical.

\section{Ensayo de tracción}

En Ingeniería la aplicación del ensayo de tracción es muy útil puesto que nos proporciona información acerca de las propiedades mecánicas, para esto se emplea una probeta normalizada, que es sujeto a un esfuerzo axial de tracción creciente hasta la ruptura de la probeta. Por medio del ensayo se conoce la resistencia de un material montada en la Máquina Universal o a una fuerza estática, dichas deformaciones son generalmente muy pequeñas, también las velocidades de deformación durante la ejecución del ensayo .

\section{Resultados.}

\section{Consideraciones del molde}

La pieza a obtenerse al tener una configuración geométrica similar a un cilindro de dimensiones pequeñas, se emplea un molde sin mazarota puesto que el mismo bebedero cumple dicha función en este caso y además el sistema de colado es directo o por lluvia.

Por otra parte, el pistón obtenido presenta un volumen de $39,38 \mathrm{~cm}^{3}$ y una área de sección transversal de $19,29 \mathrm{~cm}^{2}$; además, la base inferior del bebedero tiene un diámetro de 2,2 $\mathrm{cm}$ y su altura de $15 \mathrm{~cm}$.

\section{Tiempo de solidificación}

Aplicando la ecuación 5 se puede estimar que:

$$
\begin{gathered}
T_{T S}=C m\left(\frac{V}{A}\right)^{2} \\
T_{T S}=2\left(\frac{39,38}{19,29}\right)^{2} \\
T_{T S}=8,33
\end{gathered}
$$

Se requiere 8,33 minutos para la solidificación del pitón.

\section{Bebedero}

Al considerar en el presente trabajo de que el bebedero suplirá la función de mazarota es importante determinar que el tiempo de solidificación del bebedero que será un $40 \%$ 
adicional del tiempo de solidificación de la pieza, para disminuir el riesgo de contracción que ocurre en el proceso de solidificación de la aleación.

$$
\begin{gathered}
T_{B}=1,4 T_{T S} \\
T_{B}=11,66
\end{gathered}
$$

Por lo que el tiempo de solidificación del bebedero $\left(T_{B}\right)$, se tarda 11,66 minutos, seguidamente se estima el volumen necesario que contendrá el bebedero mediante la ecuación 5, considerando que el diámetro de la base inferior es de $2,2 \mathrm{~cm}$, y se tiene que:

$$
\begin{gathered}
V_{B}=A \sqrt{\frac{T_{B}}{C m}} \\
V_{B}=3,80 \sqrt{\frac{11,66}{2}} \\
V_{B}=9,17
\end{gathered}
$$

Resultando un volumen necesario de $9,17 \mathrm{~cm}^{3}$, por consiguiente, se tiende a calcular el Área superior del bebedero para lo cual se aplica la ecuación 1, de la manera que se tiene:

$$
\begin{gathered}
\frac{A_{1}}{A_{2}}=\sqrt{\frac{h_{1}}{h_{2}}} \\
A_{1}=3,80 \sqrt{\frac{21.31}{6.31}} \\
A_{1}=6,98
\end{gathered}
$$

Se puede determinar que requiere una superficie de $6,98 \mathrm{~cm}^{2}$, por lo que el diámetro del bebedero será de $2,98 \mathrm{~cm}$.

\section{Otros cálculos}

Con los datos obtenidos se puede determinar la velocidad de vertido en el fondo del bebedero, que se limita a la ecuación 2, y se tiene:

$$
\begin{gathered}
v=\sqrt{2 g h} \\
v=\sqrt{2 * 9,81 * 0,15} \\
v=1,72 \mathrm{~m} / \mathrm{s}
\end{gathered}
$$

El gasto volumétrico cumple la ecuación 3 y se obtiene que:

$$
\begin{gathered}
Q=v_{1} A_{1} \\
Q=10320 * 3,80
\end{gathered}
$$


Se determina que el gasto volumétrico es de $39216 \mathrm{~cm}^{3} / \mathrm{min}$, y mediante el dato obtenido se puede determinar el tiempo de vaciado que está sujeta a la ecuación 4 , que se expresa.

$$
\begin{gathered}
T_{M F}=\frac{V}{Q} \\
T_{M F}=\frac{10320}{39216} \\
T_{M F}=0,26
\end{gathered}
$$

De esta manera se puede estimar que se ha empleado 0,26 minutos (15,79 segundos) en el proceso de vaciado de la colada.

\section{Ensayos de espectrometría}

\section{Pistón original}

Como se observa en la gráfica 2 el ejemplar original contiene 54,32\% de aluminio y 29,49 $\%$ de silicio, elementos que están presentes en la aleación y que son objetos del presente

\begin{tabular}{|c|c|c|c|c|c|}
\hline \multicolumn{6}{|c|}{ Sample: } \\
\hline & Si [\%] & $\mathrm{Fe}[\%]$ & Cu [\%] & Mn [\%] & Mg [\%] \\
\hline \multirow[t]{2}{*}{$\varnothing$} & $\sim 29,49$ & 1,898 & $\sim 5,657$ & 0,349 & $\sim 3,302$ \\
\hline & $\mathrm{Cr}[\%]$ & Ni [\%] & Zn [\%] & $\mathrm{Ti}[\%]$ & $\mathrm{Pb}$ [\%] \\
\hline \multirow[t]{2}{*}{$\varnothing$} & $>0,144$ & 0,362 & 1,300 & 0,152 & $>0,264$ \\
\hline & Sn [\%] & V [\%] & Sr [\%] & $\mathrm{Zr}$ [\%] & Co [\%] \\
\hline \multirow[t]{2}{*}{$\varnothing$} & $\sim 0,376$ & $>0,108$ & 0,054 & 0,074 & 0,155 \\
\hline & $\mathrm{Ca}[\%]$ & Al [\%] & & & \\
\hline$\varnothing$ & $>0,096$ & 54,32 & & & \\
\hline
\end{tabular}
estudio, estos datos son los que generalmente se hallan con los pistones comercializados en el mercado, muy escasos hoy en día.

Gráfico 2: Espectrometría del pistón Fuente: Autor

\section{Pistón obtenido}

En la grafico 3 se muestra un contenido en la aleación de 74,47\% de aluminio y 13,64\% de silicio, porcentajes en masa, que se generó en base a la materia prima reciclada, que se obtuvo en el pistón fundido. 


\begin{tabular}{|c|c|c|c|c|c|}
\hline \multicolumn{6}{|c|}{ Bruker Analysis Report } \\
\hline \multicolumn{6}{|c|}{ Sample: } \\
\hline \multicolumn{2}{|c|}{ Analysis Time: } & \multicolumn{2}{|l|}{ 06.02.2020 16:58:53 } & \multicolumn{2}{|l|}{ Method: Al120 } \\
\hline & Si [\%] & $\mathrm{Fe}[\%]$ & $\mathrm{Cu}[\%]$ & $\mathrm{Mn}[\%]$ & $\mathrm{Mg}[\%]$ \\
\hline \multirow[t]{2}{*}{$\varnothing$} & 23,64 & 0,705 & 0,212 & 0,065 & 0,135 \\
\hline & $\mathrm{Cr}[\%]$ & $\mathrm{Ni}[\%]$ & $\mathrm{Zn}[\%]$ & $\mathrm{Ti}[\%]$ & $\mathrm{Pb}[\%]$ \\
\hline \multirow[t]{2}{*}{$\varnothing$} & 0,066 & 0,082 & 0,176 & 0,106 & 0,128 \\
\hline & Sn [\%] & $\mathrm{V}[\%]$ & $\mathrm{Sr}[\%]$ & $\mathrm{Zr}[\%]$ & Co [\%] \\
\hline \multirow[t]{2}{*}{$\varnothing$} & 0,076 & 0,014 & $<0,0030$ & 0,0056 & $<0,0030$ \\
\hline & $\mathrm{Ca}[\%]$ & Al [\%] & & & \\
\hline$\varnothing$ & 0,013 & 74,57 & & & \\
\hline
\end{tabular}

Gráfico 3: Espectrometría del pistón

Fuente: Autor

\section{Ensayo de dureza}

Para el desarrollo del ensayo de dureza se realiza la toma de 5 datos para tener confiabilidad en el resultado final, a continuación, se describen para cada uno.

\section{Pistón original}

\begin{tabular}{ccccc}
\multicolumn{5}{c}{ Tabla 1: Dureza Brinell del pistón original } \\
\hline No. & Diámetro & Unidad & Dureza & Unidad \\
\hline 1 & 0,8 & $\mathrm{~mm}$ & 363 & $H B$ \\
2 & 0,8 & $\mathrm{~mm}$ & 363 & $H B$ \\
3 & 0,8 & $\mathrm{~mm}$ & 363 & $H B$ \\
4 & 0,8 & $\mathrm{~mm}$ & 363 & $H B$ \\
5 & 0,8 & $\mathrm{~mm}$ & 363 & $H B$ \\
\hline \multicolumn{5}{c}{ Elaborado por: Autor }
\end{tabular}

Fuente: Autor

El pistón original al tratarse de un material cuya dureza es homogénea en todos los puntos se obtiene una dureza promedio de $363 \mathrm{Kg} / \mathrm{mm}^{\wedge} 2$.

\section{Pistón obtenido}

Tabla 2: Dureza Brinell del pistón obtenido

\begin{tabular}{ccccl}
\hline $\mathbf{N}^{\mathbf{0}}$. & Diámetro & Unidad & Dureza & Unidad \\
\hline 1 & 1 & $\mathrm{~mm}$ & 229 & $\mathrm{Kg}$ \\
& & & & $/ \mathrm{mm}^{2}$ \\
2 & 0,975 & $\mathrm{~mm}$ & 241 & $\mathrm{Kg}$ \\
& & & & $/ \mathrm{mm}^{2}$ \\
\hline
\end{tabular}




\begin{tabular}{|c|c|c|c|c|}
\hline 3 & 0,950 & $\mathrm{~mm}$ & 255 & $\begin{array}{l}\mathrm{Kg} \\
/ \mathrm{mm}^{2}\end{array}$ \\
\hline 4 & 1 & $\mathrm{~mm}$ & 229 & $\begin{array}{l}\mathrm{Kg} \\
/ \mathrm{mm}^{2}\end{array}$ \\
\hline 5 & 0,975 & $\mathrm{~mm}$ & 241 & $\begin{array}{l}\mathrm{Kg} \\
/ \mathrm{mm}^{2}\end{array}$ \\
\hline
\end{tabular}

La dureza ha tenido una variación en las diferentes probetas obtenidas del proceso de fundición por molde de arena verde, sin embargo, con los datos obtenidos se tiene una dureza promedio de $239 \mathrm{Kg} / \mathrm{mm}^{2}$.

\section{Ensayo de metalografía}

Para la interpretación del ensayo de metalografía se estimó los porcentajes de fase presentes en la aleación, tomando en cuenta que el porcentaje de Silicio se ubica hacia la derecha del punto eutéctico, por lo que se considera que en la muestra obtenida las fases presentes son silicio proeutectico rodeado de una matriz aluminio - silicio eutéctico.

Aplicando la regla de la palanca al diagrama de fases Al - Si del grafico 1, la cantidad de fases presentes se aproxima como se muestra a continuación.

$$
\begin{gathered}
\% S i_{\text {proeut }}=\frac{23,64-12,6}{100-12,6} * 100=12,63 \% \\
\%(A l+S i)_{\text {eut }}=100-13.03=87,37 \% \\
\left(S i_{\text {eut }} S i_{\text {proeut }}\right)=(23,64-1,6) /(100-1,6) \\
S i_{\text {eut }} S i_{\text {proeut }}=22,40 \% \\
A l_{\text {eut }}=100-22,40=77,6(\text { Al Primario }) \\
S i_{\text {eut }}=\left(S i_{\text {eut }} S i_{\text {proeut }}\right)-\% \text { Si } i_{\text {proeut }} \\
S i_{\text {eut }}=22,40-12,63=9,77 \%
\end{gathered}
$$

Con la ayuda de un software CAD, se procede a calcular la cantidad de fases presentes en función de las áreas que ocupan dentro de la muestra fotografiada, dando como resultado que el contenido de silicio proeutèctico es del $11.6999 \%$. Este valor se aproxima al valor calculado mediante regla de la palanca, con lo cual se verifica dicho resultado.

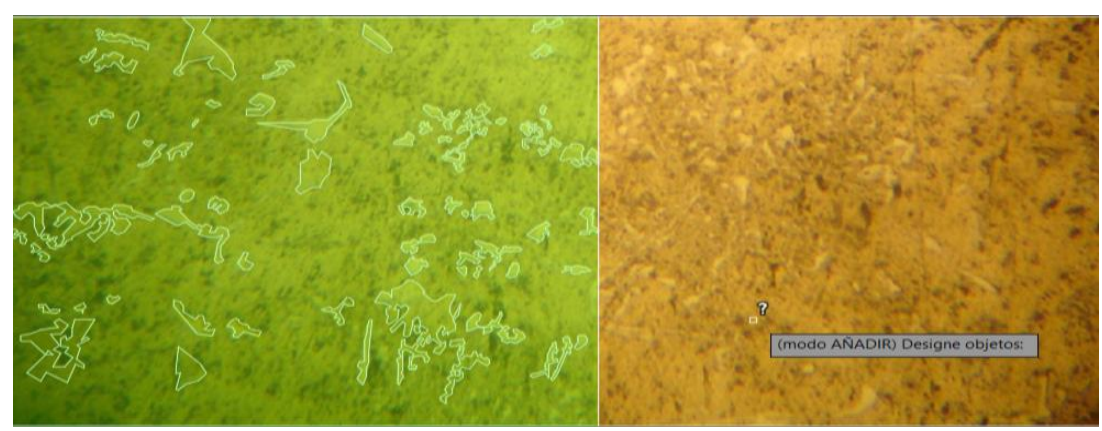

Figura 7: cantidad de fases presentes en función de las áreas

Fuente: Autor 


\section{Ensayo de tracción}

El ensayo de tracción se lo realizó solo con el material fundido mediante el proceso antes mencionado, para obtener el dato de límite de esfuerzo de la aleación resultante, la gráfica 3 proporciona información que se detallan en la tabla 3.

\section{ENSAYO DE TRACCION}

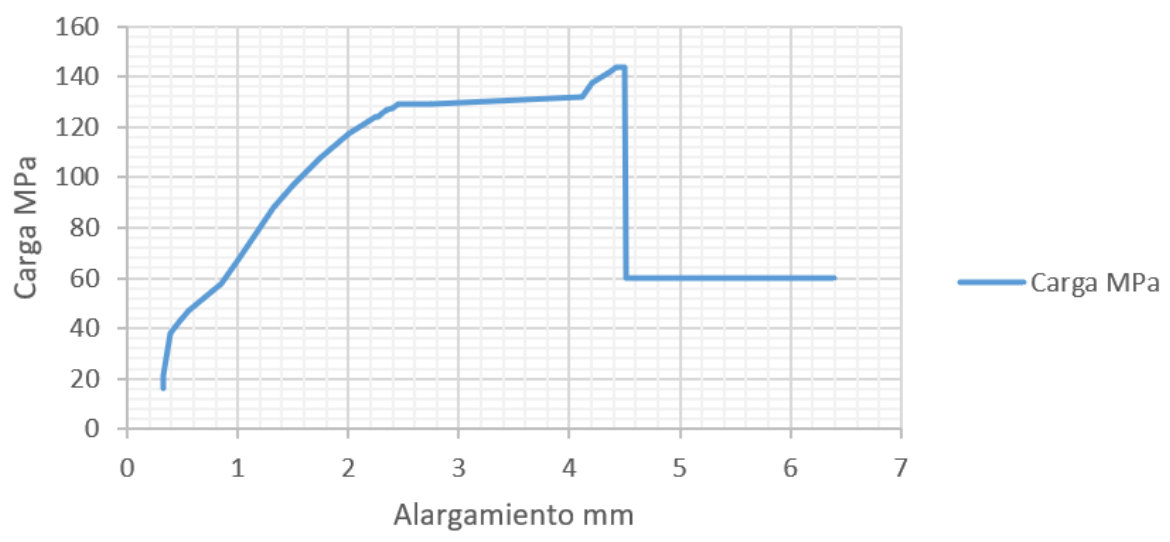

Grafica 3: Curva de ensayo de tracción de la probeta de aleación del pistón obtenido Fuente: Autor

A continuación, se presenta la tabla de resumen en la cual se detalla toda la información obtenida con el presente estudio.

Tabla 3: Datos comparativos entre el piston obtenido y original

\begin{tabular}{|c|c|c|}
\hline Ensayo & Pistón Obtenido & Pistón Original \\
\hline Espectrometría & $\begin{array}{l}\% \mathrm{Al}=74,57 \\
\% \mathrm{Si}=23,64 \\
\% \mathrm{Fe}=0,705 \\
\% \mathrm{Cu}=0,212\end{array}$ & $\begin{array}{l}\% \mathrm{Al}=54,32 \\
\% \mathrm{Si}=29,49 \\
\% \mathrm{Fe}=1,898 \\
\% \mathrm{Cu}=5,657\end{array}$ \\
\hline Dureza Brinell & $239 H B$ & $363 H B$ \\
\hline Tracción & $\begin{array}{l}\text { Desplazamiento: } 1,58 \mathrm{~mm} \\
\text { Límite de fluencia: } 129,36 \mathrm{MPa} \\
\text { Esfuerzo ultimo de tracción: } \\
143,73 \mathrm{MPa}\end{array}$ & $\begin{array}{l}\text { Desplazamiento: } \mathrm{mm} \\
\text { Límite de fluencia: MPa } \\
\text { Esfuerzo ultimo de tracción: MPa } \\
\text { - }\end{array}$ \\
\hline Espectrometría & $\begin{array}{l}\text { Se considera que en la muestra } \\
\text { obtenida las fases presentes son } \\
\text { silicio proeutéctico rodeado de una } \\
\text { matriz aluminio - silicio eutéctico. }\end{array}$ & $\begin{array}{l}\text { Se considera que en la muestra } \\
\text { original se tiene varias fases } \\
\text { intermetálicas en la matriz de } \\
\text { aluminio silicio. }\end{array}$ \\
\hline
\end{tabular}

\section{Elaborado por: Autor}

Fuente: Autor

\section{Conclusiones.}

- En el modelado es importante considerar una sobremedida en las superficies externas como margen de seguridad, para contrarrestar efectos de contracción del 
metal cuando este solidifica y se enfría a temperatura ambiente, además con la intención de dar un buen acabado superficial en el proceso de mecanizado de la pieza obtenida, esto tras el proceso de fundición.

- Mediante el empleo de los diferentes ensayos y tras conocer las aleaciones del ejemplar original, se determina que durante el proceso de fundición por molde de arena la acción de la temperatura sobre los elementos constituyentes tiende a evaporarse, así mismo, durante el vaciado de la colada en el molde de arena, las cavidades pueden generan pequeñas turbulencias mismo que se nota la presencia del fenómeno de la porosidad.

- Caracterizando mediante los diferentes ensayos en el pistón obtenido, se determina que este presenta variación relativamente baja en las propiedades específicas, tales como una disminución en la dureza y un aumento en el coeficiente de fricción.

- Con el proceso de fundición realizado se puede mencionar que la materia prima a partir del reciclado disminuye los costos de fabricación, puesto que se aprovecha la mayor parte de los elementos constituyentes, además, contribuye con el medio ambiente reduciendo el impacto ambiental y promoviendo a nuevas visiones.

\section{Referencias bibliográficas.}

ASTM E8 / E8M - 21, 2014. Standard Test Methods for Tension Testing of Metallic Materials. Part 6: Test Specimens

ASTM E10 - 18, 2017 Standard Test Method for Brinell Hardness of Metallic Materials. Part 3: Terminology and Equations

ASTM E415 - 17, 2017. Standard Test Method for Analysis of Carbon and Low-Alloy Steel by Spark Atomic Emission Spectrometry. Part 9: Preparation of Specimens and Reference Materials

ASKELAND, Donal; et al. The Science and Engineering of Materials [en línea]. 7a ed. Boston-USA: Cengage Learning INC, 2016. [Consulta: 12 abril 2020]. Disponible en:

https://books.google.com.ec/books?id=-

KOaBAAAQBAJ\&printsec $=$ frontcover\&dq $=$ The+Science+and+Engineering + of + Materials\&hl=es419\&sa=X\&ved=2ahUKEwjytbnI1I_vAhUyo1kKHQ6MBIgQ6AEwAHoECA QQAg\#v=onepage $\& \mathrm{q}=\mathrm{The} \% 20$ Science $\% 20$ and $\% 20$ Engineering $\% 20 \mathrm{of} \% 20 \mathrm{Mate}$ rials $\& f=f a l s e$

ASM INTERNATIONAL HANDBOOK COMMITTEE. Metallography and Microstructures Handbook [en línea]. 9a ed. USA: ASM International, 2004. [Consulta: 15 abril 2020]. Disponible en: https://www.asminternational.org/documents/10192/1849770/06044G_Frontmat ter.pdf 
ASM INTERNATIONAL HANDBOOK COMMITTEE. Properties and selection: Nonferrous alloys and special-purpose materials [en línea]. 2a ed. USA: ASM International, 1993. [Consulta: 07 mayo 2020]. Disponible en: http://sme.vimaru.edu.vn/sites/sme.vimaru.edu.vn/files/volume_2__properties_and_selection_nonf.pdf

CAO, Xinjin \& CAMPBELL, John. "The nucleation of Fe-Rich phases on oxide films in Al11.5Si0.4Mg cast alloys". Metall mater trans a [en línea], 2003, (Canada). [Consulta: 20 julio 2020]. ISSN 1090-7807. Disponible en: https://www.researchgate.net/publication/248131734_The_nucleation_of_FeRich_phases_on_oxide_films_in_Al115Si04Mg_cast_alloys

DAVIS, Joseph. Aluminum and Aluminum Alloys [en línea]. 6a ed. USA: ASM International, 2007. [Consulta: 22 abril 2020]. Disponible en: https://books.google.com.ec/books?id=Lskj5k3PSIcC\&printsec $=$ frontcover\&dq $=$ Aluminum + and + Aluminum + Alloys $\& \mathrm{hl}=\mathrm{es}-419 \& \mathrm{sa}=\mathrm{X} \& \mathrm{ved}=2 \mathrm{ahUKEwjrvK}-$ U3I_vAhXDq1kKHWhrD_UQ6AEwAHoECAIQAg\#v=onepage \&q=Aluminu $\% 20$ and $\% 20$ Aluminum $\% 20$ Alloys $\& \mathrm{f}=$ false

MACKAY, R. "Quantification of iron in aluminium-silicon foundry alloys via thermal analysis". International Journal of Cast Metals Research [en línea], 2016, (London). [Consulta: 20 agosto 2020]. ISSN 1090-7807. Disponible en: https://www.tandfonline.com/doi/abs/10.1080/13640461.1997.11819228

ROMMING, C; et al. "Crystal structure of p-AI4.5FeSi". Acta Crystallographica Section B [en línea], 1993, (Great Britain). [Consulta: 20 agosto 2020]. ISSN 1090-7807. Disponible en: http://scripts.iucr.org/cgi-bin/paper?S0108768193013096

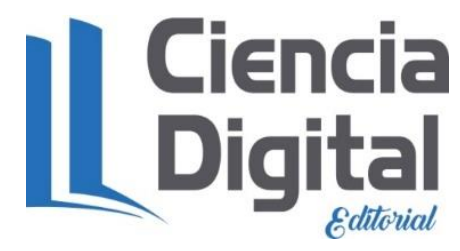


PARA CITAR EL ARTÍCULO INDEXADO.

Abarca Pérez, E. P., Bravo Morocho, V. D., Cayancela Achote, J. L., \& Buenaño Moyano, L. F. (2021). Desarrollo de un proceso de obtención de un pistón de $100 \mathrm{cc}$ de dos tiempos mediante ingeniería inversa. AlfaPublicaciones, 3(3), 78-97. https://doi.org/10.33262/ap.v3i3.70

\section{Liencia}

El artículo que se publica es de exclusiva responsabilidad de los autores y no necesariamente reflejan el pensamiento de la Revista Alfa Publicaciones.

El artículo queda en propiedad de la revista y, por tanto, su publicación parcial y/o total en otro medio tiene que ser autorizado por el director de la Revista Alfa Publicaciones.
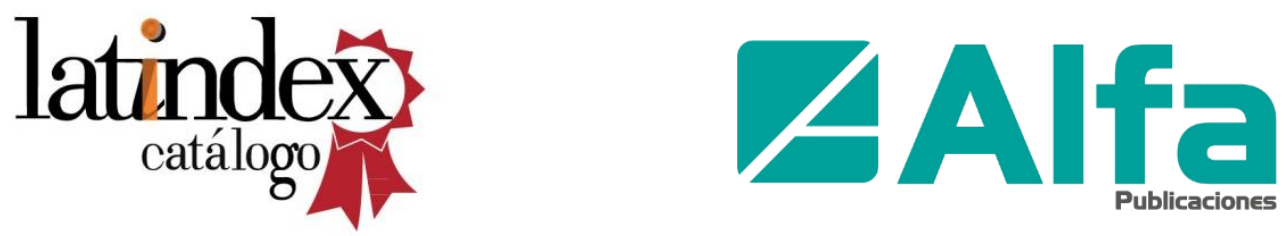